\title{
Prognostic value of different cardiac magnetic resonance imaging derived parameters in Egyptian patients with ST- elevation myocardial infarction after successful reperfusion by primary percutaneous intervention
}

\author{
Diaa Kamal ${ }^{1 *}$ D, Ahmed S. Ibrahim², Merhan Ahmed Nasr $^{2}$ and Sherihan S. Madkour²
}

\begin{abstract}
Background: Cardiac magnetic resonance (CMR) is an extremely accurate and useful modality that can give much data about myocardial damage after acute myocardial infarction and consequently can give a good idea about long-term prognosis. Unfortunately, this modality is still underused in Egypt. We tried to assess the prognostic significance of different parameters derived from CMR in Egyptian patients presenting with ST-elevation myocardial infarction (STEMI) treated by primary percutaneous intervention (PPCI). Twenty-five patients who presented with acute STEMI and were successfully reperfused by PPCI within $12 \mathrm{~h}$ from symptoms onset were included. CMR was performed 2-4 days after PPCl. Six months of long-term follow-up for major adverse cardiovascular events (reinfarction, new-onset heart failure and cardiac death) was done. CMR-derived parameters (edema volume, area at risk, infarction volume, infarction percentage, microvascular obstruction volume, microvascular obstruction percentage, myocardial salvage and myocardial salvage index) were analyzed in relation to incidence of major adverse cardiovascular events (MACE).

Results: Seven patients suffered from MACE. Univariate logistic regression analysis showed a significant correlation between edema volume $(P=0.04)$, area at risk $(P=0.05)$, infarction percentage $(P=0.05)$ and the occurrence of MACE. Multivariate logistic regression analysis showed that infarction percentage $(P=0.05)$ is the best parameter that can predict MACE.

Conclusion: Infarction percentage is potentially the most important prognosticator derived from CMR in Egyptian patients with acute STEMI successfully reperfused by PPCI.
\end{abstract}

Keywords: ST-elevation myocardial infarction, Primary percutaneous coronary intervention, Cardiac magnetic resonance, Major adverse cardiovascular events

\footnotetext{
* Correspondence: drdiaaeldin@med.asu.edu.eg

${ }^{1}$ Cardiology department, Faculty of medicine, Ain Shams University, Abbasia

Street, Cairo, Egypt

Full list of author information is available at the end of the article
} 


\section{Background}

Ischemic heart disease (IHD) is the leading cause of death worldwide and also in Egypt $[1,2]$. Deaths due to coronary artery disease (CAD) reached $24.58 \%$ of total deaths in Egypt according to the most recent data from the World Health Organization (WHO) [1]. In the last decade, a good decline in short- and long-term mortality after STelevation myocardial infarction (STEMI) is obvious owing to the extended use of primary percutaneous coronary intervention (PPCI), thrombolytic therapies, modern antithrombotics and measures of secondary prevention [3]. Management of STEMI patients during this period is also improving in Egypt with an increase in the number of patients treated with PPCI yielding results that can be compared to Western data [4].

However, this favorable decline is simultaneously generating an increasing cohort of STEMI survivors who are prone to long-term mechanical and arrhythmic hazards [5]. Identification of high-risk STEMI patients is very important to risk stratify them and properly incorporate them into secondary prevention programs to avoid complications. Up till now, all practice guidelines utilize post-MI echocardiography-derived left ventricular ejection fraction (EF) as the most important measure for risk stratification of STEMI patients [6, 7]. Echocardiography in the context of acute MI has important limitations related to myocardial stunning and compensatory hyperkinesia of healthy myocardium that can make its value as an important prognosticator debatable [8].

Cardiac magnetic resonance (CMR) is an imaging modality that is gaining an increasing interest from investigators in the field of post-STEMI risk stratification owing to its ability to accurately describe and quantify the irreversible damage that affected the infarcted myocardium and consequently predict adverse clinical outcomes beside its ability to evaluate ventricular functions and morphology [9].

Unfortunately, till now there is no consensus about which CMR-derived parameter can be used as the best prognosticator that can be used accurately to risk stratify patients after STEMI.

In our small study, we tried to get preliminary data about the prognostic significance of different CMRderived parameters in Egyptian patients presenting with STEMI treated by PPCI.

\section{Methods}

This study was a prospective observational study that was conducted upon 25 patients presented to the cardiology department in Ain Shams University hospitals in the time interval from August 2016 to January 2017.

The study was approved by the Research Ethics Committee (Faculty of Medicine, Ain Shams University, FWA 00006444) and all patients signed an informed consent for participation in the study in accordance with the
Declaration of Helsinki. We included patients presented with acute STEMI (any territory) within the first $12 \mathrm{~h}$ after onset of chest pain who were candidates for PPCI according to the most recent practice guidelines $[6,7]$. There was no age or sex predilection.

\section{Exclusion criteria}

Patients with delayed presentation ( $>12 \mathrm{~h}$ ), any previous history of ischemic heart diseases, hemodynamic instability, renal impairment (serum creatinine $>2 \mathrm{mg} / \mathrm{dl}$ ), contraindications to MRI (claustrophobia, an implanted metallic device, pacemakers ...), pregnant and lactating females were excluded from the study.

Eligible patients presented with acute STEMI were directly transferred with close monitoring to the catheterization laboratory after doing a rapid thorough clinical examination and signing an informed consent. Rapid evaluation with bedside echocardiography to exclude mechanical complications was done for all patients. All patients received an oral loading dose of Aspirin $300 \mathrm{mg}$ and Clopidogrel $600 \mathrm{mg}$. Coronary angiography was done rapidly by operators highly experienced in PPCI using adequate orthogonal views to detect the culprit lesion. PPCI was done aiming at flow restoration in the culprit artery. Patients were then transferred to coronary care unit (CCU) under close monitoring and were kept on anti-ischemic measures.

All relevant characteristics of our patients were recorded including demographic characteristics, presence of major cardiovascular risk factors, myocardial territory affected, Killip class on presentation [10], procedural variables (thrombolysis in myocardial infarction [TIMI] flow before and after the procedure [11], use of thrombectomy device, method of revascularization [PTCA or stenting], type of stents, number of stents, myocardial blush grade [MBG] [12] after revascularization and presence of other major non-culprit artery stenosis) and postprocedural variables (peak cardiac enzymes, echocardiography variables and short-term complications related to MI or PCI procedure).

CMR was done 2-4 days after PPCI at the Radiodiagnosis Department, Ain Shams University using a 1.5 Tesla superconductive MR scanner (Philips Achieva-XR Medical Systems, Best, the Netherlands) with dedicated phased array Cardiac coil for enhanced resolution and increased signal-to-noise ratio.

The basic protocol included were single-shot black blood (axial, sagittal and coronal views), Cine short axial views, Cine 4 chamber views, Dynamic fast field echo (FFE) and Lock Locker.

The main sequences that were interpreted in this study were:

(a) T2WI STIR (TR 2 beats, TE 80) (to detect the edematous changes in the form of bright signal intensity changes) 
(b) Post-contrast imaging: gadolinium was administered as bolus of $0.1 \mathrm{mmol} / \mathrm{kg}$ then delayed imaging from 10 to $30 \mathrm{~min}$ according to lock locker. (To detect the ischemic territorial affection, infarct size, percentage of microvascular occlusion).

In patients with microvascular obstruction (MO), the dark areas were included within infarct size analysis and the area of MO was evaluated separately.

Then the following parameters were calculated:

- Area at risk = edema volume/volume of left ventricle (LV) mass.

- Percentage of infarct size (infarction fraction) = infarction volume/volume LV mass.

- Percentage of $\mathrm{MO}=$ volume $\mathrm{MO} /$ volume LV mass.

- Myocardial salvage $=$ area at risk - infarct size .

- Myocardial salvage index (MSI) = area at risk infarct size/area at risk.

The primary endpoint of the study was the occurrence of any major adverse cardiovascular events (MACE) defined as death or re-infarction or new-onset heart failure at 6 months follow-up after the index event.

\section{Statistical analysis}

Data were analyzed using MedCalc@ version 15 (MedCalcœ Software bvba, Ostend, Belgium) and version 23

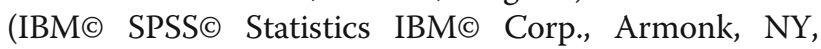
USA) and normality of numerical data distribution was examined using the D'Agostino-Pearson test. Nonnormally distributed numerical variables were presented as median and interquartile range and between-group differences were compared by the Mann-Whitney test. Categorical variables were presented as the percentage and number. $P$ value was used to determine the statistical significance. A value of 0.05 is arbitrary a cut-off value; below it, the relationship between variables is considered statistically significant, and above it, the relation is considered statistically not significant.

\section{Results}

\section{Demographic and clinical characteristics}

The studied population included $20(80 \%)$ males. The median age group was $48.3 \pm 8.6$ years. Seventeen (68\%) were smokers, $4(16 \%)$ had a history of Tramadol drug addiction, $2(8 \%)$ had a history of cannabis smoking. Eight (32\%) were diabetics, 4 (16\%) were hypertensive, 2 (8\%) had dyslipidemia and 3(12\%) had a family history of premature CAD. Regarding Killip class on presentation, $23(92 \%)$ cases were classified as Killip class I, $2(8 \%)$ were classified as Killip class II while none of our study population was presented with Killip class III or IV. ECG done on presentation showed $3.8 \pm 1.8 \mathrm{~mm}$ ST- segment elevation with $1.8 \pm 1.0 \mathrm{~mm}$ reciprocal STsegment depression. Demographic and clinical data together with time intervals of clinical relevance are shown in [Table 1].

\section{Primary PCI procedural variables}

Analysis of procedural variables showed that LAD was the culprit artery in $20(80 \%)$ cases, RCA in $3(12 \%)$ of cases and LCX in $2(8 \%)$ of cases. Twenty-two (88\%) patients had TIMI 0 flow in the culprit artery and $3(12 \%)$ patients had TIMI 1 flow. Restoration of flow was done using PTCA in $15(60 \%)$ patients and thrombus aspiration devices in $7(28 \%)$ patients. Only one $(4 \%)$ patient did not receive a stent, 21 (84\%) patients received one stent and $3(12 \%)$ patients received 2 stents. Only 2 DES were implanted and all other stents were BMS. All procedures ended up with regaining TIMI 3 flow. Fourteen (56\%) patients had postprocedural MBG III in the affected territory, while 11 (44\%) patients had MBG II. All PPCI procedural data are shown in [Table 2].

\section{Postprocedural variables}

In-hospital echocardiographic and CMR variables are shown in [Table 3]. Examples from our cases are shown in [Figs. 1 and 2]. Short-term MACE was encountered in the form of early stent thrombosis in 2 patients $(8 \%)$ and anticoagulant-related hemorrhage which occurred in

\begin{tabular}{ll} 
Table 1 Demographic and clinical data of our study population \\
\hline Age (years) & $48.3 \pm 8.6(30-65)$ \\
Male gender & $20(80 \%)$ \\
Smoking & $17(68 \%)$ \\
Tramadol addiction & $4(16 \%)$ \\
Cannabis addiction & $2(8 \%)$ \\
DM & $8(32 \%)$ \\
Hypertension & $4(16.0 \%)$ \\
Dyslipidemia & $2(8.0 \%)$ \\
Obesity & $2(8.0 \%)$ \\
Old DVT & $1(4 \%)$ \\
Family history of premature CAD & $3(12 \%)$ \\
Killip I & $23(92.0 \%)$ \\
Killip II & $2(8 \%)$ \\
ST elevation (mm) & $3.8 \pm 1.8$ \\
Reciprocal ST depression (mm) & $1.8 \pm 1$ \\
Pain-to-door time (hours) & $5.6 \pm 2.7$ \\
Door-to-balloon time (min.) & $52.8 \pm 67.2$ \\
Pain-to-balloon time (hours) & $6.1 \pm 3.6$ \\
Total pain time (hours) & $6.9 \pm 3.5$ \\
Time to peak cardiac enzyme level (hours) & $13.7 \pm 5.0$ \\
Pain-to-ST-segment resolution (hours) & $7.8 \pm 4.7$ \\
\hline &
\end{tabular}


Table 2 Primary $\mathrm{PCl}$ variables

\begin{tabular}{|c|c|}
\hline \multicolumn{2}{|l|}{ Restoration of flow } \\
\hline No PTCA & $9(36 \%)$ \\
\hline PTCA & $15(60 \%)$ \\
\hline Wire only & $1(4 \%)$ \\
\hline Thrombus aspiration & $7(28 \%)$ \\
\hline \multicolumn{2}{|l|}{ Number of stents } \\
\hline Nil & $1(4 \%)$ \\
\hline One & $21(84 \%)$ \\
\hline Two & $3(2 \%)$ \\
\hline \multicolumn{2}{|l|}{ Stent type } \\
\hline BMS & $22(88 \%)$ \\
\hline DES & $2(8 \%)$ \\
\hline \multicolumn{2}{|l|}{ TIMI flow before $\mathrm{PCl}$} \\
\hline TIMI 0 & $22(88 \%)$ \\
\hline TIMI I & $3(12 \%)$ \\
\hline \multicolumn{2}{|l|}{ TIMI flow after PCI } \\
\hline TIMI III & $25(100 \%)$ \\
\hline \multicolumn{2}{|l|}{ MBG post-PCl } \\
\hline Grade II & $11(44 \%)$ \\
\hline Grade III & $14(56 \%)$ \\
\hline \multicolumn{2}{|l|}{ Thrombus grading } \\
\hline Grade III & $4(16 \%)$ \\
\hline Grade IV & $1(4 \%)$ \\
\hline Grade V & $20(80 \%)$ \\
\hline \multicolumn{2}{|l|}{ Culprit vessel } \\
\hline LAD & $20(80 \%)$ \\
\hline LCX & $2(8 \%)$ \\
\hline $\mathrm{RCA}$ & $3(12 \%)$ \\
\hline \multicolumn{2}{|l|}{ Non-culprit vessel } \\
\hline LAD & $2(8 \%)$ \\
\hline LCX & $4(16 \%)$ \\
\hline $\mathrm{RCA}$ & $5(20 \%)$ \\
\hline D1 & $1(4 \%)$ \\
\hline
\end{tabular}

only one patient (4\%). Long-term 6 months follow-up for the occurrence of MACE showed that 7 (28\%) patients were affected. One patient suffered from reinfarction with acute heart failure, 3 patients suffered from re-infarction and 3 patients suffered from manifestations of heart failure.

Correlation between clinical, demographic, periprocedural characteristics and incidence of MACE

Apart from DM which showed a significant correlation with the incidence of MACE $(P=0.02)$, all other demographic, clinical and periprocedural variables did not show any significant relationship with this incidence.
Table 3 In-hospital echocardiographic and CMR variables

\begin{tabular}{ll}
\hline Echocardiography & $6(24 \%)$ \\
Normal diastolic functions & $10(40 \%)$ \\
DD Grade I & $9(36 \%)$ \\
DD Grade II & $2(8 \%)$ \\
LV thrombus formation & $46 \pm 9$ \\
EF on day 2 (\%) & \\
CMR & $68,063.0 \pm 34$, \\
Volume of myocardial edema (mm ${ }^{3}$ ) & 057.0 \\
Area at risk (fraction of LV mass) & $0.419 \pm 0.21$ \\
Infarction volume (mm ${ }^{3}$ ) & $27,455 \pm 22,094$ \\
Infarcted fraction (fraction of area at risk) & $0.163 \pm 0.112$ \\
MO volume (mm ${ }^{3}$ ) & $4058.0 \pm 6271.0$ \\
MO fraction (fraction of LV mass) & $0.022 \pm 0.033$ \\
Myocardial salvage fraction (fraction of & $0.255 \pm 0.197$ \\
area at risk) & \\
MSI & $0.582 \pm 0.275$ \\
LV cross-sectional area by echocardiography & $1916.6 \pm 359.7$ \\
(mm²) & \\
LV cross-sectional area by MRI (mm²) & $4451.5 \pm 2875.0$ \\
EF on day 2 by MRI (\%) & $52.0 \pm 11$ \\
\hline
\end{tabular}

\section{Correlation between CMR-derived parameters and incidence of MACE}

Univariate analysis showed a significant correlation between edema volume, area at risk, infarction percentage and occurrence of MACE with $P$ values of 0.04, 0.05 and 0.05 respectively. On the contrary, MSI failed to prove a significant correlation with MACE. Stepwise multivariate backward logistic regression analysis showed that infarction percentage is the most important CMR-derived parameter that can predict the occurrence of $\operatorname{MACE}(P=$ 0.05).

\section{Discussion}

Management of patients with acute STEMI had enormously improved in the last decade and primary PCI became the standard of care for those patients $[6,7]$. Despite this improvement in standards of care, mortality is still more than 7\% during the first month after STEMI but progressively improves, although still considerable afterwards [5]. Cost-effective methods for early risk stratification are extremely needed for patient management by detection of high-risk patients and thereby incorporating those patients in short- and long-term follow-up programs to improve morbidity and mortality. CMR is one of these methods that is extremely appealing but unfortunately underused in Egypt mainly due to limited resources. 

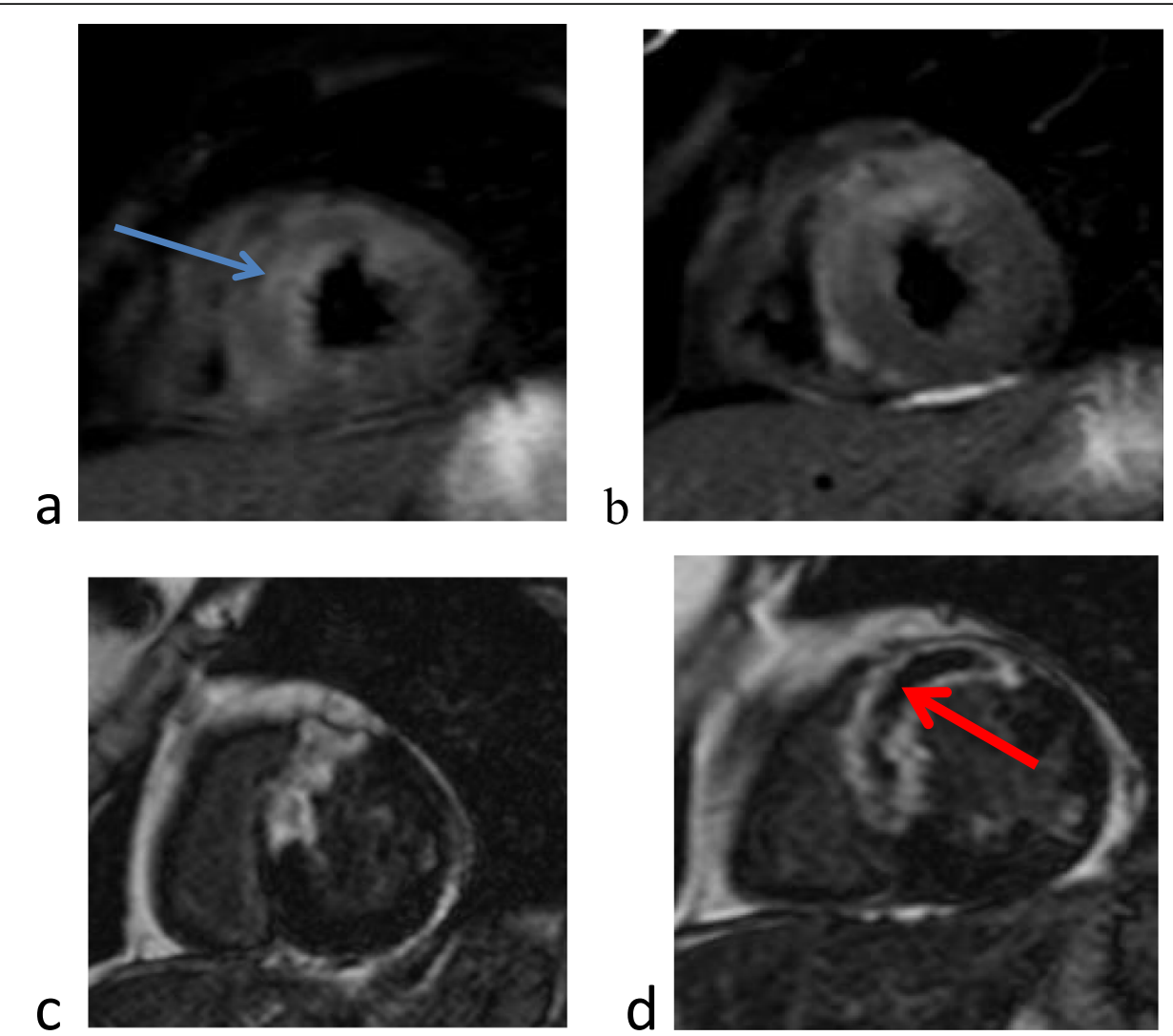

Fig. 1 Case number 7 presented with anterior STEMI: a, b short-axis T2STIR images reveals edematous changes $\{b l u e$ arrow\} involving LAD territory $\mathbf{c}$, d short-axis delayed post-contrast images reveals transmural infarction involving LAD territory with large central MO \{red arrow\}

In this small study, we tried to start highlighting the role of different CMR-derived prognosticators in the management of this cohort of patients in Egypt. Our study was an observational prospective pilot study that included 25 patients who suffered an acute STEMI and were treated by PPCI. After 6 months of follow-up, 7 (28\%) patients developed MACE.

We found that infarction percentage is the most important CMR-derived predictor for the occurrence of MACE. The amount of myocardium that is finally jeopardized by the acute ischemic insult has a direct impact on LV systolic functions which is up till now, the most important predictor of poor outcomes in this category of patients according to the most recent international guidelines [6,7].

Our results in this context are agreeing with the results of the study done by Stiermaier et al. who used 2 separate patients' cohorts to derive and validate a CMRderived risk score to predict MACE in patients with acute STEMI treated by PPCI at 12 months interval. Infarction percentage $\geq 19 \%$ of LV size was one of the 3 components of this score [13].

MSI failed to prove a significant correlation with MACE in our study population. This was in contrary to the results obtained by Eitel et al., who showed that MSI is a strong predictor of MACE in these patients [14]. This difference might be attributed to the higher incidence of anterior STEMI in our study (80\%) versus (47\%) in their study. Patients with anterior STEMI can develop more complications even with a higher MSI due to the presence of a large affected territory. Also, the small number of our study population might have contributed to this discordance.

$\mathrm{MO}$ volume and percentage failed to prove any significant correlation with MACE in our study population. Our results in this context were different from those obtained by Symons et al. in their study which showed that MO detected by CMR at a median of 4 days after the index event is a strong predictor of poor outcomes in STEMI patients revascularized by PPCI, they also concluded that MO extent $\geq 2.6 \%$ of LV mass is a strong independent predictor of MACE [9]. Also, MO $\geq 1.4 \%$ of LV was one of the 3 components of the risk score developed and validated by Stiermaier et al. in their study [13]. In their study, Eitel et al. showed a similar significant predictive value of $\mathrm{MO} \geq 1.4 \%$ of LV [15]. Again, Van Kranenburg et al. showed in their meta-analysis that included $>1000$ patients who suffered STEMI in 8 CMR studies that MO can predict MACE independently [16]. 
a

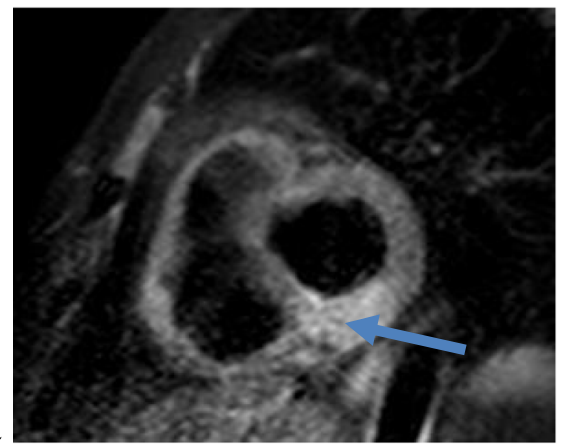

c

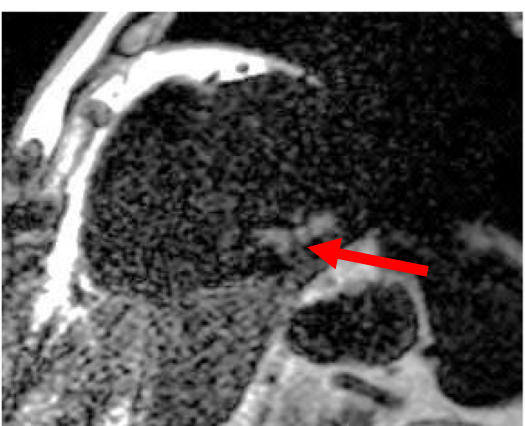

$\mathrm{b}$

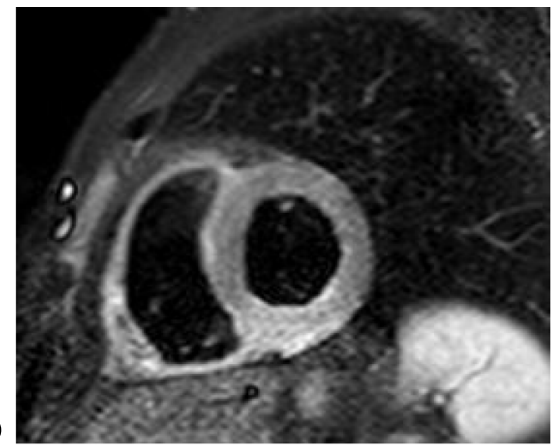

d

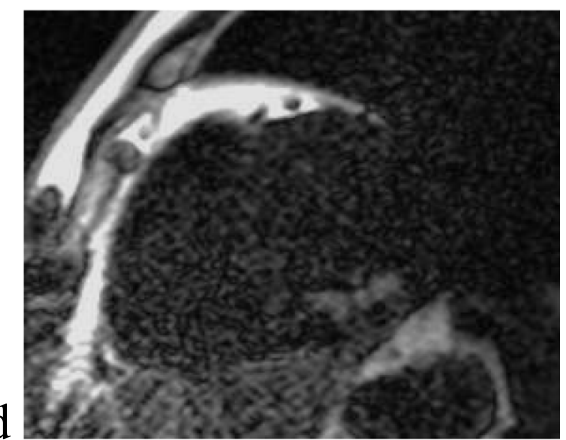

Fig. 2 Case number 8 presented with inferior STEMI: a, b short-axis T2STIR images reveals edematous changes involving RCA territory $\{$ blue arrow\}, edema volume $58,538 \mathrm{~mm}^{3} \mathbf{c}$, $\mathbf{d}$ short-axis delayed post-contrast images reveals transmural infarction involving RCA territory with no MO rred arrow\}

Many studies were performed to understand the correlation between ischemic preconditioning, $\mathrm{MO}$ and its long-term effect after the acute event [17-19]. Many patients in developing countries experience prolonged ischemic preconditioning prior to developing an acute coronary syndrome more than their counterparts in developed countries due to lack of medical awareness and this might have decreased the significance of MO in our study versus other studies done in European and American centers. Also, there is no standard algorithm for LGE and MO quantification in STEMI and different protocols are used in different studies. Again, the small number of our study population might have affected our results.

Our study limitations were that our sample size was small; this made us unable to exclude the effect of other confounding factors on the occurrence of MACE. Our study was a single-center study and all our patients were Caucasian. Patients with cardiogenic shock and hemodynamic instability were excluded from our study. Longer follow-up intervals might be needed in future studies. Future studies are also needed in which PPCI is performed using DES only and preferably eluting the same drug from the same stent platform.

\section{Conclusions}

Our study showed that infarction percentage is potentially the most important CMR-derived prognosticator in Egyptian patients with acute STEMI successfully reperfused by PPCI. It is also evident that despite having many studies in the past few years trying to figure out important CMR-derived parameters that affect prognosis in this category of patients, none of these studies gave solid data about which parameter is most important in prediction of outcome and there is no consensus till now about the most efficient prognosticator. Consequently; up till now, nothing of these parameters was incorporated in risk stratification models issued in the most recent guidelines of management for patients with STEMI [6, 7]. Rapid improvements in the technology, decreasing cost and standardization of study protocols will certainly pave the road for CMR to be included in future guidelines.

\footnotetext{
Abbreviations

CAD: Coronary artery diseases; CMR: Cardiac magnetic resonance; EF: Ejection fraction; IHD: Ischemic heart disease; LGE: Late gadolinium enhancement; LV: Left ventricle; MACE: Major adverse cardiovascular events; MBG: Myocardial blush grade; MI: Myocardial infarction; MO: Microvascular obstruction; MSI: Myocardial salvage index; PPCI: Primary percutaneous coronary intervention; STEMI: ST-segment elevation myocardial infarction; TIMI: Thrombolysis in myocardial infarction; WHO: World Health Organization
}

Acknowledgements

Technicians and nursing teams of the Cardiology and Radio-diagnosis departments, Ain Shams University hospitals. 


\section{Authors' contributions}

DK participated in the study design and coordination, performed the primary $\mathrm{PCl}$ procedures, followed up patients and drafted the manuscript. ASI participated in the study design and coordination participated in performance and analysis of CMR studies. MAN participated in performance and analysis of CMR studies. SSM participated in performance and analysis of CMR studies, followed up patients and performed data collection and statistical analysis. All authors read and approved the final manuscript.

\section{Funding}

We did not receive any specific fund to cover this research

\section{Availability of data and materials}

The datasets used and analyzed during the current study are available from the corresponding author on reasonable request.

\section{Ethics approval and consent to participate}

The study was approved by the Research Ethics Committee (Faculty of Medicine, Ain Shams University, FWA 00006444) and all patients signed an informed consent for participation in the study in accordance with the Declaration of Helsinki.

\section{Consent for publication}

Not applicable.

\section{Competing interests}

The authors declare that they have no competing interests.

\section{Author details}

'Cardiology department, Faculty of medicine, Ain Shams University, Abbasia Street, Cairo, Egypt. ${ }^{2}$ Radiodiagnosis Department, Faculty of medicine, Ain Shams University, Cairo, Egypt.

Received: 24 May 2019 Accepted: 3 December 2019

Published online: 21 December 2019

\section{References}

1. The top 10 causes of death worldwide: WHO January 2017 update. Available at http://www.who.int/mediacentre/factsheets/fs310/en/. Accessed Apr 2019

2. World Health Rankings (2017) Available at http://www.worldlifeexpectancy. com/egypt-coronary-heart-disease. Accessed Apr 2019

3. Townsend N, Wilson L, Bhatnagar P et al (2016) Cardiovascular disease in Europe: epidemiological update 2016. Eur Heart J 37(42):3232-3245

4. Sobhy M, Sadaka M, Okasha N et al (2012) Stent for life initiative placed at the forefront in Egypt 2011. Eurolntervention 8:108-115

5. Pedersen F, Butrymovich V, Kelbæk H et al (2014) Short- and long-term cause of death in patients treated with primary PCI for STEMI. JACC 64: 2101-2108

6. Ibanez B, James S, Agewall S et al (2017) 2017 ESC guidelines for the management of acute myocardial infarction in patients presenting with STsegment elevation. Eur Heart J 00:1-66

7. Levine GN, Bates ER, Blankenship JC et al (2016) 2015 ACC/AHA/SCAI focused update on primary percutaneous coronary intervention for patients With ST-elevation myocardial infarction. JACC 67(10):1235-1250

8. Califf RM, Harrelson-Woodlief L, Topol EJ (1990) Left ventricular ejection fraction may not be useful as an end point of thrombolytic therapy comparative trials. Circulation 82:1847-1853

9. Symons R, Pontone G, Schwitter J et al (2018) Long-term incremental prognostic value of cardiovascular magnetic resonance after ST-segment elevation myocardial infarction. A study of the collaborative registry on CMR in STEMI. JACC Cardiovasc Imaging 11(6):813-825

10. Killip T, Kimball JT (1967) Treatment of myocardial infarction in a coronary care unit. A two year experience with 250 patients. Am J Cardiol 20:457-464

11. Rind $E$, Arbel $Y$, Finkelstein A et al (2009) Angiographic evaluation of epicardial and microvascular coronary flow. IMAJ 11:173-177

12. Webb I, Redwood S (2007) Measuring reperfusion in the catheter laboratory Heart Metab 37:9-13

13. Stiermaier T, Jobs A, De Waha S et al (2017) Optimized prognosis assessment in ST-segment-elevation myocardial infarction using a cardiac magnetic resonance imaging risk score. Circ Cardiovasc Imaging 10: e006774. https://doi.org/10.1161/CIRCIMAGING. 117.006774

14. Eitel I, Desch S, Fuernau G et al (2010) Prognostic significance and determinants of myocardial salvage assessed by cardiovascular magnetic resonance in acute reperfused myocardial infarction. JACC 55(22):2470-2479

15. Eitel I, de Waha S, Wöhrle J et al (2014) Comprehensive prognosis assessment by CMR imaging after ST-segment elevation myocardial infarction. JACC 64:1217-1226

16. Van Kranenburg M, Magro M, Thiele $\mathrm{H}$ et al (2014) Prognostic value of microvascular obstruction and infarct size, as measured by CMR in STEMI patients. JACC Cardiovasc Imaging 7:930-939

17. Posa A, Pavo N, Hemetsberger R et al (2010) Protective effect of ischaemic preconditioning on ischaemia/reperfusion induced microvascular obstruction determined by online measurements of coronary pressure and blood flow in pigs. Thromb Haemost 103(02):450-460

18. Jesel L, Morel O, Ohlmann P et al (2007) Role of pre-infarction angina and inflammatory status in the extent of microvascular obstruction detected by MRI in myocardial infarction patients treated by PCI. Int J Cardiol 121(2): 139-147

19. Sloth AD, Schmidt MR, Munk K et al (2014) Improved long-term clinical outcomes in patients with ST-elevation myocardial infarction undergoing remote ischaemic conditioning as an adjunct to primary percutaneous coronary intervention. Eur Heart J 35(3):168-175

\section{Publisher's Note}

Springer Nature remains neutral with regard to jurisdictional claims in published maps and institutional affiliations.

\section{Submit your manuscript to a SpringerOpen ${ }^{\circ}$ journal and benefit from:}

- Convenient online submission

- Rigorous peer review

- Open access: articles freely available online

High visibility within the field

- Retaining the copyright to your article

Submit your next manuscript at $\boldsymbol{\nabla}$ springeropen.com 